\title{
An Observer-Based Finite Control Set Model Predictive Control for Three-Phase Power Converters
}

\author{
Tao Liu, ${ }^{1}$ Changliang Xia, ${ }^{1,2}$ Xin Gu, ${ }^{2}$ and Tingna Shi ${ }^{1}$ \\ ${ }^{1}$ School of Electrical Engineering and Automation, Tianjin University, Tianjin 300072, China \\ ${ }^{2}$ Tianjin Key Laboratory of Advanced Technology of Electrical Engineering and Energy, Tianjin Polytechnic University, \\ Tianjin 300387, China \\ Correspondence should be addressed to Changliang Xia; motor@tju.edu.cn
}

Received 3 April 2014; Accepted 22 May 2014; Published 7 July 2014

Academic Editor: Piermarco Cannarsa

Copyright (C) 2014 Tao Liu et al. This is an open access article distributed under the Creative Commons Attribution License, which permits unrestricted use, distribution, and reproduction in any medium, provided the original work is properly cited.

\begin{abstract}
Finite control set model predictive control (FCS-MPC) for three-phase power converters uses a discrete mathematical model of the power converter to predict the future current value for all possible switching states. The circuit parameters and measured input currents are necessary components. For this reason, parameter error and time delay of current signals may degrade the performance of the control system. In the previous studies of the FCS-MPC, few articles study these aspects in detail and almost no method is proposed to avoid these negative influences. This paper, first, investigates the negative impacts of inductance inaccuracy and ACside current distortion due to the time delay caused by filter on FCS-MPC system. Then, it proposes an observer-based FCS-MPC approach with which the inductance error can be corrected, the current signal's time delay caused by filter can be compensated, and therefore the performance of FCS-MPC will be improved. At last, as an example, it illustrates the effectiveness of the proposed approach with experimental testing results for a power converter.
\end{abstract}

\section{Introduction}

Power converters based on pulse-width modulation (PWM) technology have been widely applied in modern industry field [1-3]. PWM converters are becoming more and more popular in recent years. Comparing with the traditional diode-based rectifiers, the PWM converters have many advantages such as unity power factor, bidirectional power flow, and low harmonic distortion [4]. Finite control set model predictive control (FCS-MPC) is an alternative control strategy for power converters and drives. This method has developed rapidly in the past few years [5-8]. Taking into account the discrete nature of power converters, FCS-MPC predicts the future behaviors for all possible switching states over a time frame (finite sampling instants), and the optimal one that minimizes the cost function is selected to obtain the desired output. FCS-MPC has attracted experimental applications due to its advantages such as flexibility to control different variables, inherent decoupling behavior, fast dynamic response, and easy inclusion of nonlinearities and constraints [9-14].
In FCS-MPC based system, parameter uncertainty and measurement noise may affect the behaviors of the control system. The negative influences of the errors of equivalent series resistance and input inductance have been addressed in several publications $[15,16]$, but extensive studies on this issue were not performed and method to avoid the negative influence introduced by parameter error has not been addressed so far.

The classical FCS-MPC algorithm only chooses one switching state in each sampling period, so the switching frequency is not constant. Although the transient response is better, it demands high sampling frequency and the impact of control system time delay is more obvious on the operating performance. In practical applications, time delays can be caused by signal measurement, filtering, calculation, and actuation. Among them the calculation and filtering have an obvious impact on the performance of the control system. A two-step-ahead prediction has been proposed to compensate the calculation time delay $[15,17,18]$. Article [18] provides a clear and detailed explanation of the two-stepahead prediction. 


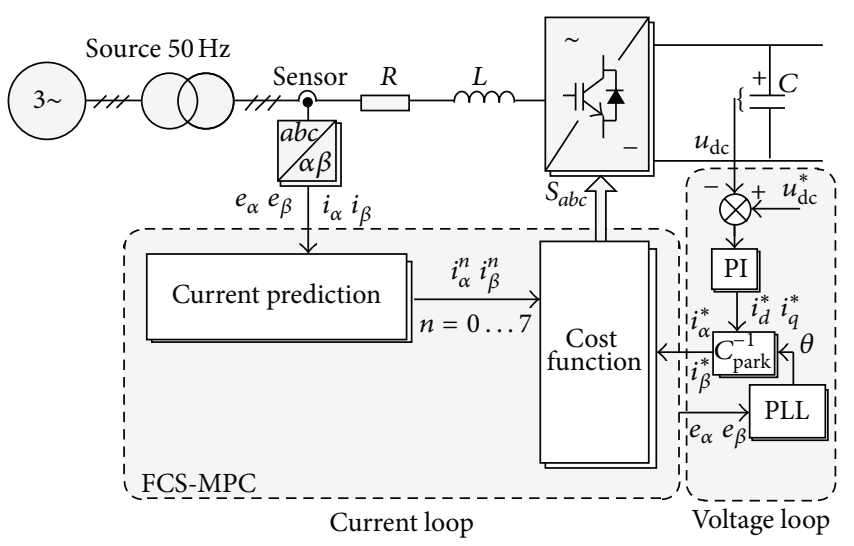

FIGURE 1: Block diagram of the FCS-MPC based power converter.

Filtering is another factor which will cause the time delay of the control system. To remove the unwanted highfrequency noise, the current signals need to be filtered before the A/D conversion. The signal filtering introduces time delay and causes deviation on sampling signals, which consequently cause a high current ripple of the power converter. Finally, the performance of the system is significantly deteriorated.

Although the calculation time delay has been discussed in some articles, the time delay caused by filter almost has not been discussed in the FCS-MPC strategy. In the classical linear control methods for power converters, such as PI current controllers with pulse-width modulation based methods, the filter time delay can be solved by adjusting the parameters in the PI or other linear controllers, but, in FCSMPC system, the situation is different. The FCS-MPC is a nonlinear control algorithm without modulation, so it has no parameters similar with those in PI controller. For this reason, it is necessary to find a new method to avoid the negative influence caused by filter time delay.

In this paper, the impacts of the input inductance error and filter time delay are analyzed in detail, respectively. A novel observer-based FCS-MPC strategy is proposed in the 2-level power converter. This algorithm can compensate ACside inductance error and time delay caused by the filter. Finally, experimental results are presented to validate the theoretical studies.

\section{Operating Principle of Classical FCS-MPC}

Like classical control strategy of power converters, the FCSMPC based power converter, as shown in Figure 1, has two control loops. The voltage control loop includes a PI controller to minimize the difference between the output DC bus voltage and its reference, while the current control loop is based on FCS-MPC strategy, which keeps the $\alpha \beta$ axis currents tracking its references. In the controller, the reference angle of the source voltage can be estimated by the phase-locked-loop (PLL).

Below begins with the development of the discrete-time model of a power converter circuit, shown in Figure 2, and

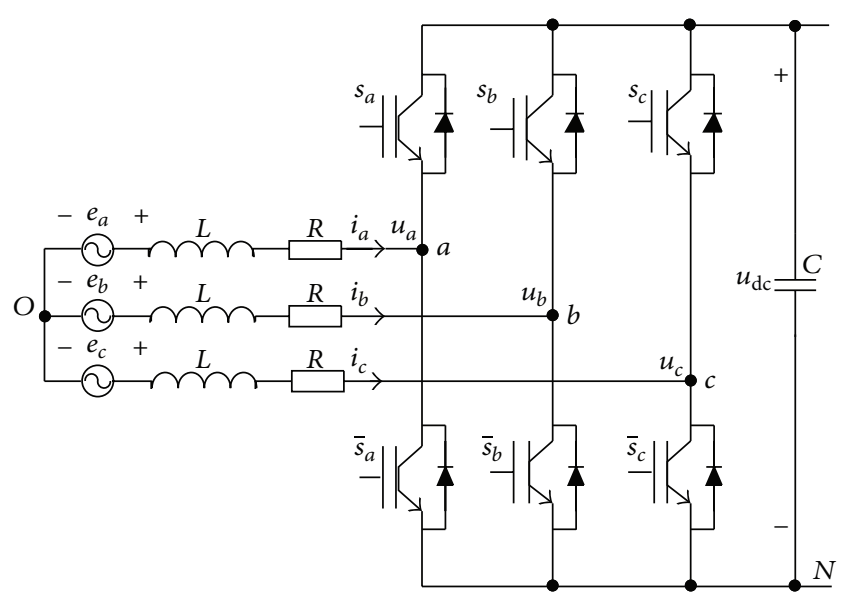

FIGURE 2: The topological structure of main circuit of a 2-level power converter.

then brings up the factors which affect the performance of corresponding FCS-MPC strategy.

According to the circuit topology of the 2-level power converter shown in Figure 2, the voltage equations of the 2level power converter can be written as

$$
\begin{aligned}
& L \frac{\mathrm{d} i_{a}}{\mathrm{~d} t}=e_{a}-u_{a}-R i_{a}, \\
& L \frac{\mathrm{d} i_{b}}{\mathrm{~d} t}=e_{b}-u_{b}-R i_{b}, \\
& L \frac{\mathrm{d} i_{c}}{\mathrm{~d} t}=e_{c}-u_{c}-R i_{c},
\end{aligned}
$$

where $i_{a}, i_{b}$, and $i_{c}$ are the input currents; $e_{a}, e_{b}$, and $e_{c}$ are the source voltages; $u_{a}, u_{b}$, and $u_{c}$ are the converter voltages; $R$ is the equivalent series resistance; and $L$ is the AC-side inductance.

Performing Clarke transformation on (1), the voltage equations in stationary $\alpha-\beta$ reference frame can be obtained as

$$
\begin{aligned}
& L \frac{\mathrm{d} i_{\alpha}}{\mathrm{d} t}=e_{\alpha}-u_{\alpha}-R i_{\alpha}, \\
& L \frac{\mathrm{d} i_{\beta}}{\mathrm{d} t}=e_{\beta}-u_{\beta}-R i_{\beta},
\end{aligned}
$$

where $i_{\alpha}$ and $i_{\beta}$ are the input currents; $e_{\alpha}$ and $e_{\beta}$ are the source voltages; and $u_{\alpha}, u_{\beta}$ are the converter voltages.

Using the finite difference approximation, the derivative term $\mathrm{d} i / \mathrm{d} t$ in (2) can be expressed by

$$
\frac{\mathrm{d} i}{\mathrm{~d} t} \approx \frac{i(k+1)-i(k)}{t(k+1)-t(k)}=\frac{i(k+1)-i(k)}{T},
$$

where $i(k+1)$ and $i(k)$ are the current at time instant $t(k+1)$ and $t(k)$, respectively. $T$ is the sampling time of the current control loop. 
TABLE 1: Voltage space vectors.

\begin{tabular}{|c|c|c|c|}
\hline$n$ & $s_{a}$ & $\begin{array}{ll}s_{b} & s_{c} \\
\end{array}$ & {$\left[\begin{array}{ll}u_{\alpha}^{n} & u_{\beta}^{n}\end{array}\right]$} \\
\hline 0 & 0 & 00 & {$[0,0]$} \\
\hline 1 & 1 & 00 & {$\left[2 u_{\mathrm{dc}} / 3,0\right]$} \\
\hline 2 & & 10 & {$\left[-u_{\mathrm{dc}} / 3, \sqrt{3} u_{\mathrm{dc}} / 3\right]$} \\
\hline 3 & 1 & 10 & {$\left[u_{\mathrm{dc}} / 3, \sqrt{3} u_{\mathrm{dc}} / 3\right]$} \\
\hline 4 & & $\begin{array}{ll}0 & 1\end{array}$ & {$\left[-u_{\mathrm{dc}} / 3,-\sqrt{3} u_{\mathrm{dc}} / 3\right]$} \\
\hline 5 & & $\begin{array}{ll}0 & 1\end{array}$ & {$\left[u_{\mathrm{dc}} / 3,-\sqrt{3} u_{\mathrm{dc}} / 3\right]$} \\
\hline 6 & 0 & 11 & {$\left[-2 u_{\mathrm{dc}} / 3,0\right]$} \\
\hline 7 & & 11 & {$[0,0]$} \\
\hline
\end{tabular}

Substituting (3) into (2) and rearranging the equations give the discrete-time expression for currents in the power converter

$$
\begin{aligned}
& i_{\alpha}(k+1)=\frac{T}{L}\left[e_{\alpha}(k)-u_{\alpha}(k)-R i_{\alpha}(k)\right]+i_{\alpha}(k), \\
& i_{\beta}(k+1)=\frac{T}{L}\left[e_{\beta}(k)-u_{\beta}(k)-R i_{\beta}(k)\right]+i_{\beta}(k) .
\end{aligned}
$$

From (4), then, the formula used for current prediction can be obtained as

$$
\begin{array}{r}
i_{\alpha}^{n}(k+1)=\frac{T}{L}\left[e_{\alpha}(k)-u_{\alpha}^{n}(k)-R i_{\alpha}(k)\right]+i_{\alpha}(k), \\
i_{\beta}^{n}(k+1)=\frac{T}{L}\left[e_{\beta}(k)-u_{\beta}^{n}(k)-R i_{\beta}(k)\right]+i_{\beta}(k) \\
n=0,1, \ldots, 7,
\end{array}
$$

where $n$ denotes the switching state of IGBTs, which has eight options; $u_{\alpha}^{n}(k)$ and $u_{\beta}^{n}(k)$ are the converter's input voltages at time instant $k$, under IGBT switching state $n ; i_{\alpha}^{n}(k+1)$ and $i_{\beta}^{n}(k+1)$ are the input currents predicted for the next time instant, $k+1$, under the same IGBT switching state $n$.

Table 1 gives the determination of $u_{\alpha}^{n}$ and $u_{\beta}^{n}$, corresponding to each IGBT switching state $n=(0, \ldots, 7)$, where $s_{a} s_{b} s_{c}$ denotes the switching signals at three IGBTs at top of power converter circuit, shown in Figure 2, while the other three IGBTs at the bottom are the inverse signals of $s_{a} s_{b} s_{c}$.

To achieve an optimal prediction, a cost function needs to be defined. The cost functions may take different forms based on different purposes [15]. Here in this paper, the summation of the square of the difference between the predicted currents and the reference currents in $\alpha$-axis and $\beta$-axis, respectively, is adopted:

$$
\begin{array}{r}
g_{n}(k+1)=\left[i_{\alpha}^{*}(k+1)-i_{\alpha}^{n}(k+1)\right]^{2} \\
+\left[i_{\beta}^{*}(k+1)-i_{\beta}^{n}(k+1)\right]^{2} \\
n=0,1, \ldots, 7,
\end{array}
$$

where $i_{\alpha}^{*}(k+1)$ and $i_{\beta}^{*}(k+1)$ are the reference currents at time instant $k+1$.

Finally, the optimal prediction is considered to be achieved by applying the corresponding switching state which gives the smallest $g_{n}(k+1)$ over the time range from $t(k)$ to $t(k+1)$.

Figure 1 and the current prediction (5) indicate that the performance of the FCS-MPC presented above depends on the accuracy of parameters (such as $L$ and $R$ ) and input currents $i_{\alpha}$ and $i_{\beta}$. L varies with the saturation status of the inductor core due to the magnetization nonlinearity; the accuracy of input currents is unavoidably affected by the signal filtering process. In the following section, impacts of parameter variation and filtering-resulted time delay on the FCS-MPC are studied.

\section{Observer-Based FCS-MPC for Power Converters}

3.1. Inductance Observer. Inductance error, discussed here, is the difference between the inductance value used by FCSMPC strategy and the actual inductance value of the physical inductor. In this paper, the inductance value used by FCSMPC strategy is called "model inductance," and the actual value of the physical inductor is called "actual inductance." Inductance error, resulted in such a way, may deteriorate the performance of FCS-MPC. Experimental results in Figure 3 are an example to illustrate this concern. It can be seen that the input current waveform distortion becomes obvious when model inductance $L_{m}$ used by the current prediction calculation becomes $40 \%$ of its actual value.

The function of the proposed inductance observer is to estimate the actual value of the inductance in the power converter.

Considering (4), one can obtain the expression of the $\alpha$ axis current at time instant $k$

$$
\begin{aligned}
i_{\alpha}(k)= & \frac{T}{L}\left[e_{\alpha}(k-1)-u_{\alpha}(k-1)-R i_{\alpha}(k-1)\right] \\
& +i_{\alpha}(k-1) .
\end{aligned}
$$

The estimated value of the inductance can be defined as $\widehat{L}$. With the estimated inductance $\widehat{L}$, the estimated current $\widehat{i}_{\alpha}(k)$ is

$$
\begin{aligned}
\hat{i}_{\alpha}(k)= & \frac{T}{\widehat{L}(k-1)}\left[e_{\alpha}(k-1)-u_{\alpha}(k-1)-R i_{\alpha}(k-1)\right] \\
& +i_{\alpha}(k-1) .
\end{aligned}
$$

Subtracting (7) from (8), one can obtain the reciprocal of actual inductance $L$ as

$$
\frac{1}{L}=\frac{1}{\widehat{L}(k-1)}+\frac{i_{\alpha}(k)-\hat{i}_{\alpha}(k)}{T\left[e_{\alpha}(k-1)-u_{\alpha}(k-1)-R i_{\alpha}(k-1)\right]} .
$$




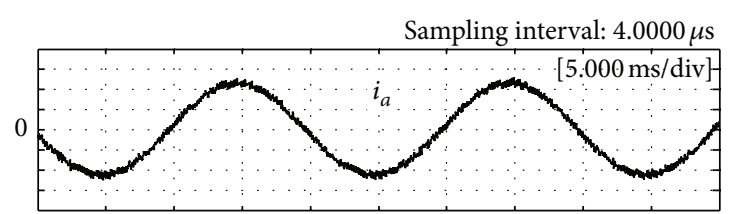

(a) Phase- $a$ current waveform (3 A/div) when $L=L_{m}=5.0 \mathrm{mH}$

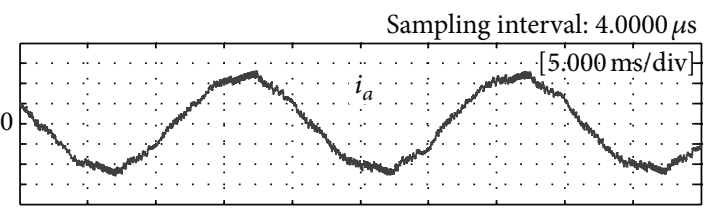

(b) Phase- $a$ current waveform ( $3 \mathrm{~A} / \mathrm{div})$ when $L=5.0 \mathrm{mH}$ and $L_{m}=2.0 \mathrm{mH}$

FIGURE 3: The negative impacts caused by inductance error in the current prediction.

With the step value $r$, the equation for estimating the value of inductance $L$ can be

$$
\begin{aligned}
& \frac{1}{\widehat{L}(k)} \\
& = \begin{cases}\frac{1}{\widehat{L}(k-1)}+r \frac{i_{\alpha}(k)-\hat{i}_{\alpha}(k)}{T\left[e_{\alpha}(k-1)-u_{\alpha}(k-1)-R i_{\alpha}(k-1)\right]} \\
\frac{1}{\widehat{1} e_{\alpha}(k-1)-u_{\alpha}(k-1)-R i_{\alpha}(k-1) \neq 0}\end{cases} \\
& \begin{array}{ll}
\text { if } e_{\alpha}(k-1)-u_{\alpha}(k-1)-R i_{\alpha}(k-1)=0 .
\end{array}
\end{aligned}
$$

Substituting (8) into (10) and rearranging the equations, the inductance observer is finally obtained as

$$
\begin{aligned}
& \frac{1}{\widehat{L}(k)} \\
& = \begin{cases}\frac{(1-r)}{\widehat{L}(k-1)}+r \frac{i_{\alpha}(k)-i_{\alpha}(k-1)}{T\left[e_{\alpha}(k-1)-u_{\alpha}(k-1)-R i_{\alpha}(k-1)\right]} \\
\text { if } e_{\alpha}(k-1)-u_{\alpha}(k-1)-R i_{\alpha}(k-1) \neq 0\end{cases}
\end{aligned}
$$

Since the $1 / \widehat{L}(k)$ can be directly applied into the current prediction in (5), the calculation for $\widehat{L}(k)$ is not needed. This will avoid the amplification of noise in the division operation. By changing the parameter $r$, the dynamic response speed of the observer can be adjusted.

3.2. Filter Time Delay Observer. FCS-MPC is a control algorithm which uses current signal to perform the current prediction then voltage vector selection for IGBT switching control. In possible future applications, the implementation of FCS-MPC requires a filtering operation on the measured current signals to ensure FCS-MPC functions as expected. In Figure 1, the filter block is usually placed in front of $a b c / \alpha \beta$ transformation block to serve such a purpose. A properly designed filter does help to take the unwanted high frequency harmonic noises off the measured current signals. However, the filtering operation results in a time delay between the current signal before filtering and the current signal after filtering. The time delay of the current signal plays a negative role in FCS-MPC. The influence of time delay distortion of currents on FCS-MPC is investigated and some results are presented below.

Figure 4(a) shows that the current signal time delay increases with the decrease of the filter cut-off frequency. The utilization of filter of lower cut-off frequency cleans the harmonics noise better, but it results in a longer time delay of the current signal, in other words, more time delay distortion of the current signal.

As a result, Figure 4(b) gives the comparison of the three-phase current waveforms obtained by using a 1-order low-pass filter of cut-off frequency of $1 \mathrm{kHz}$ and $20 \mathrm{kHz}$, respectively. It shows that the current waveform obtained with the use of $1 \mathrm{kHz}$ cut-off frequency filtering contains more low frequency harmonics than the one obtained with the use of the $20 \mathrm{kHz}$ cut-off frequency filtering. Such a result tells us that low-pass filtering removes the unwanted high frequency noises but brings in low frequency harmonics due to the time delay distortion of the current signal.

The identification of the problems promotes finding approaches to solve them. A filter time delay observer is proposed to eliminate the corresponding negative impacts of the filtering-resulted time delay on FCS-MPC. It is presented below starting with the mathematical description of the power converter circuit.

According to (2), the state equation of the power converter can be obtained as

$$
\begin{aligned}
& \frac{\mathrm{d} i_{\alpha}}{\mathrm{d} t}=-\frac{R}{L} i_{\alpha}+\frac{1}{L} e_{\alpha}-\frac{1}{L} u_{\alpha}, \\
& \frac{\mathrm{d} i_{\beta}}{\mathrm{d} t}=-\frac{R}{L} i_{\beta}+\frac{1}{L} e_{\beta}-\frac{1}{L} u_{\beta} .
\end{aligned}
$$

Taking consideration of the 1-order low-pass filter operation on the current signals, one has

$$
F(s)=\frac{I_{f}(s)}{I(s)}=\frac{1}{1+a s},
$$

where $I(s)$ is the current signal before filtering; $I_{f}(s)$ is the current signal after filtering; $F(s)$ is transfer function of filter; $a=1 /\left(2 \pi f_{c}\right)$; and $f_{c}$ is the cut-off frequency of the filter; this equation is shown in frequency domain. 


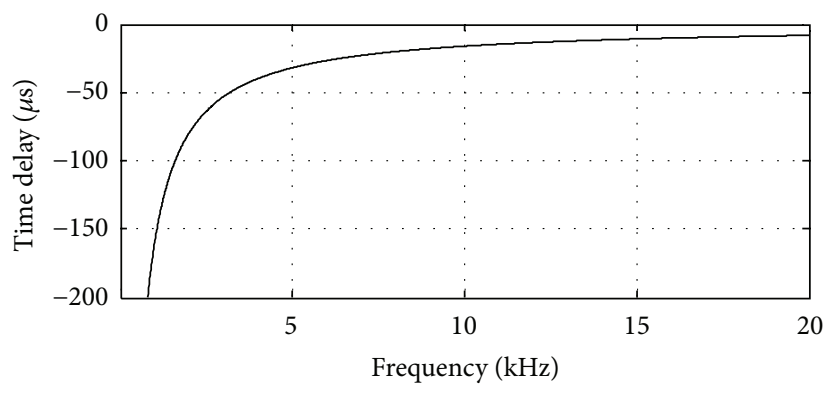

(a) Time delay depends on the cut-off frequency (1-order low-pass filter)

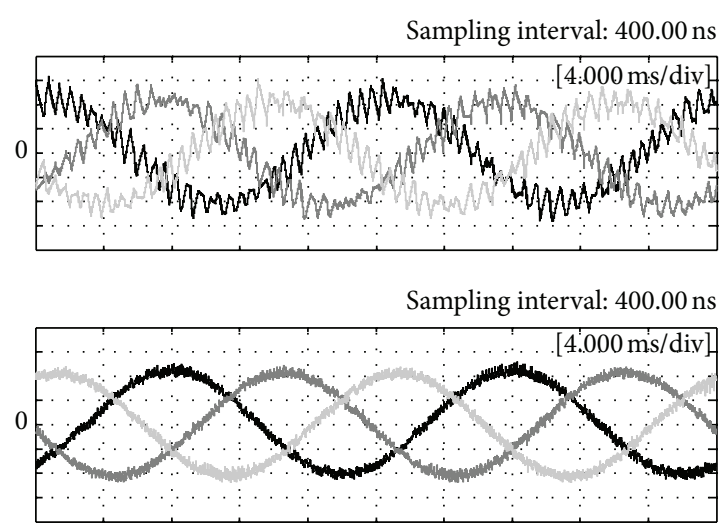

(b) The input current waveforms $(3.0 \mathrm{~A} / \mathrm{div})$ with cut-off frequency $1 \mathrm{kHz}$ (upper figure) and $20 \mathrm{kHz}$ (lower figure), respectively

FIGURE 4: The negative impacts caused by filtering-resulted time delay on FCS-MPC.

Equation (13) can be rewritten into the state equation form. Considering the current signals filtering in the FCSMPC based power converters, one has

$$
\begin{aligned}
& \frac{\mathrm{d} i_{\alpha f}}{\mathrm{~d} t}=\frac{1}{a} i_{\alpha}-\frac{1}{a} i_{\alpha f}, \\
& \frac{\mathrm{d} i_{\beta f}}{\mathrm{~d} t}=\frac{1}{a} i_{\beta}-\frac{1}{a} i_{\beta f},
\end{aligned}
$$

where $i_{\alpha}$ and $i_{\beta}$ are the current signals before filtering and $i_{\alpha f}$ and $i_{\beta f}$ are the current signals after filtering.

With the consideration of (12) and (14), the proposed filter time delay observer is presented as follows:

$$
\begin{aligned}
& \frac{\mathrm{d} \hat{i}_{\alpha}}{\mathrm{d} t}=\frac{1}{L} e_{\alpha}-\frac{1}{L} u_{\alpha}-\frac{R}{L} \widehat{i}_{\alpha}+l\left(i_{\alpha f}-\widehat{i}_{\alpha f}\right), \\
& \frac{\mathrm{d} \hat{i}_{\beta}}{\mathrm{d} t}=\frac{1}{L} e_{\beta}-\frac{1}{L} u_{\beta}-\frac{R}{L} \widehat{i}_{\beta}+l\left(i_{\beta f}-\widehat{i}_{\beta f}\right), \\
& \frac{\mathrm{d} \hat{i}_{\alpha f}}{\mathrm{~d} t}=\frac{1}{a} \hat{i}_{\alpha}-\frac{1}{a} \hat{i}_{\alpha f}, \\
& \frac{\mathrm{d} \hat{i}_{\beta f}}{\mathrm{~d} t}=\frac{1}{a} \widehat{i}_{\beta}-\frac{1}{a} \hat{i}_{\beta f},
\end{aligned}
$$

where $\hat{i}_{\alpha}$ and $\hat{i}_{\beta}$ are the estimated current signals before filtering; $\hat{i}_{\alpha f}$ and $\hat{i}_{\beta f}$ are the estimated current signals after filtering; and $l$ is the proportional parameter of the observer.

The function of the proposed filter time delay observer is to provide FCS-MPC with the currents which do not contain unwanted high frequency noise and without time delay. In other words, the purpose of the filter time delay observer is to eliminate the impacts of the current signals time delay caused by filters. The filters can also cause time delay on the source voltage signals, but the source voltages can be regarded as system disturbance variables instead of state variables of the system; the time delay on these variables $\left(e_{\alpha}\right.$ and $\left.e_{\beta}\right)$ has lower impact on the performance of the power converter, so, in this paper, the source voltage signals $e_{\alpha}$ and $e_{\beta}$ are thought to not have changed after the process of filtering.

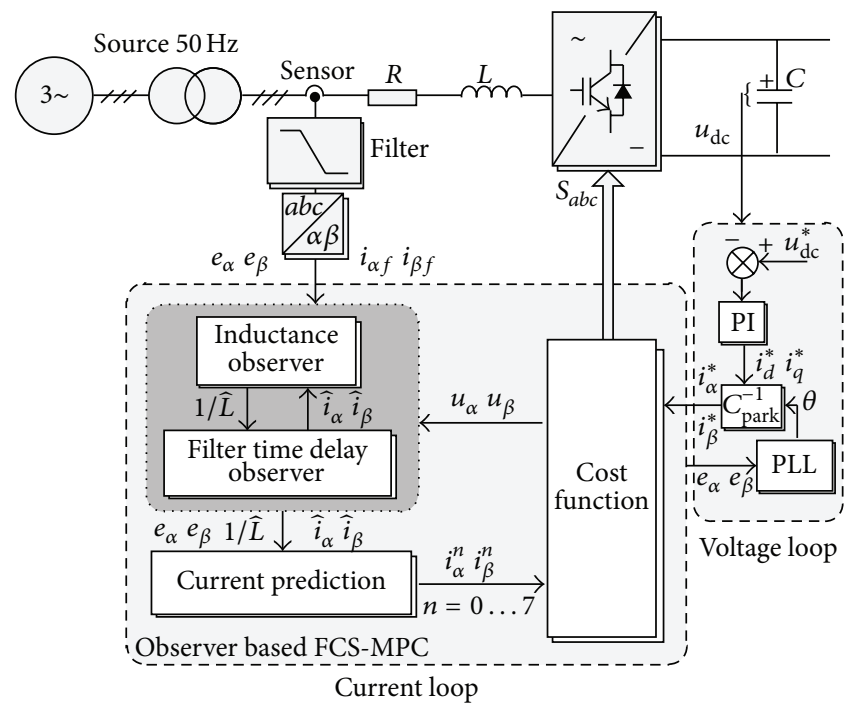

FIGURE 5: Block diagram of the proposed FCS-MPC scheme with designed observer.

3.3. Observer-Based FCS-MPC. The working principle of proposed observers and their integration with the FCS-MPC are presented in the following section.

The inductance observer and the filter time delay observer interact with each other to enhance their performance. Inductance observer uses the estimated currents $\hat{i}_{\alpha}$ and $\hat{i}_{\beta}$ (without filtering-resulted time delay) provided by the filter time delay observer to obtain a closer estimation for $L$; filter time delay observer uses $\widehat{L}$ estimated by the inductance observer to obtain currents $\hat{i}_{\alpha}$ and $\hat{i}_{\beta}$ to be more desirable for FCS-MPC.

Finally, integration of the combined observer block with the FCS-MPC of power converter shown in Figure 1 gives the control diagram of the observer-based FCS-MPC of power converter, which is shown in Figure 5. 


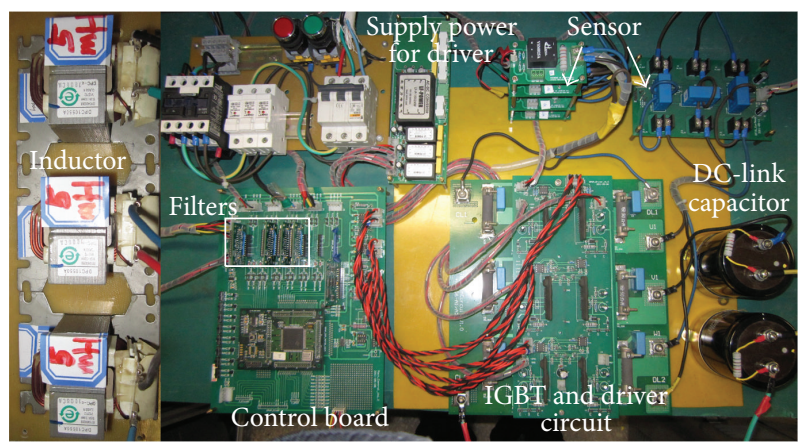

FIGURE 6: Experimental platform of the three-phase 2-level power converter.

TABLE 2: The main experiment parameters.

\begin{tabular}{lc}
\hline Parameters & Values \\
\hline Rated voltage (line-line) & $110 \mathrm{~V}(\mathrm{Rms})$ \\
Equivalent series resistance & $1.20 \Omega$ \\
AC-side inductance & $5.0 \mathrm{mH}$ \\
Load resistance & $41.4 \Omega$ \\
DC voltage & $180 \mathrm{~V}$ \\
\hline
\end{tabular}

Comparing the control diagram in Figure 5 with the one in Figure 1, one can restate the advantages of the observedbased FCS-MPC over the previous FCS-MPC. With the use of the proposed observer, FCS-MPC performs the IGBTs switching state determination by using the estimated value of inductance instead of a supposed fixed value and the input currents without unwanted noise and filtering-resulted time delay instead of currents with filtering-resulted time delay. As a result, the observer-based FCS-MPC power converter is expected to provide a better control performance than the previous FCS-MPC power converter.

The effectiveness of the proposed inductance observer and the filter time delay observer is verified by the experimental testing. Details are presented below.

\section{Experimental Verification}

An experimental platform (shown in Figure 6) was developed using TI floating point digital signal processing (DSP) TMS320F28335. The sensor signals are filtered by 1-order lowpass filter. The experimental parameters are shown in Table 2. The sampling time of the current control loop is $50 \mu \mathrm{s}$ $(T=50 \mu \mathrm{s})$.

4.1. Inductance Error Compensation. Figure 7 shows performance of the inductance observer in the FCS-MPC based power converter. To minimize the influence of the time delay caused by filters, the cut-off frequency of 1-order filter is $20 \mathrm{kHz}$.

Figure 7(a) shows the accuracy and dynamic performance of the inductance observer. The actual inductance $L$ can change rapidly between $5.0 \mathrm{mH}$ and $6.2 \mathrm{mH}$ by cutting-in or cutting-off an additional inductor $(1.2 \mathrm{mH})$. In the figure, it can be seen that the output result $\widehat{L}$ of the inductance
TABLE 3: Time dependencies for the proposed algorithm.

\begin{tabular}{lc}
\hline Module & $\begin{array}{c}\text { Execution } \\
\text { time }[\mu \mathrm{s}]\end{array}$ \\
\hline Total algorithm & 33 \\
Sampling and A/D conversion & 5 \\
FCS-MPC strategy (including voltage loop and PLL) & 12.5 \\
Calculation delay compensation & 2 \\
Proposed observer & 3.5 \\
Others (protection, DAC, etc.) & 10 \\
\hline
\end{tabular}

observer traces the actual inductance $L$ with high speed and well accuracy.

In Figure 7(b), the model inductance $L_{m}$ used by the current prediction calculation is $40 \%$ of its actual value, that is, $L_{m}=2.0 \mathrm{mH}$ and $L=5.0 \mathrm{mH}$. It can be seen that, because of the inductance error, the current distortion of $i_{a}$ is obvious. When the estimated value of the inductance observer is added into the control system, the current distortion of $i_{a}$ becomes much smaller.

4.2. Filter Time Delay Compensation. Figures 8 and 9 show the experimental performance of the filter time delay observer. The cut-off frequency of the low-pass filter is $1 \mathrm{kHz}$. The model inductance $L_{m}$ is $5.0 \mathrm{mH}$ and the same as actual value $L$ in the physical system.

In Figure 8, the power converter operates in the intense background noises environment. It can be seen that, because of the time delay caused by filter, the current distortion is more obvious. Moreover, the actual current $i_{a}$ contains a lot of high frequency noise. With the process of the filter, the current $i_{\text {af }}$ after filtering is smoother than $i_{a}$ but with the time delay, while the estimated current $\hat{i}_{a}$ is not only smooth but also with no time delay compared to the actual current $i_{a}$.

In Figures 9(a) and 9(b), it can be seen that, after the addition of the estimated results $\left(\hat{i}_{\alpha}\right.$ and $\left.\hat{i}_{\beta}\right)$ of the observer, the ripple of the currents becomes much lower. The performance of power converter system has been improved with the observer. In the experiment, the background noises have been weakened to make current waveforms easy to be distinguished.

4.3. Startup Sequence of the Observers. Figure 10 presents the current waveforms with different startup sequence of the inductance observer and filter time delay observer. It can be seen that the observers operate stably and well in the following two types of situations. This shows well flexibility and stability of the proposed observer-based FCS-MPC for power converters. The background noises have been weakened to make current waveforms easy to be distinguished.

4.4. Execution Time of the Observer. The detail execution time of the proposed observer-based FCS-MPC is given in Table 3. It can be seen that, compared with the entire execution time of the FCS-MPC algorithm, the cost time $(3.5 \mu \mathrm{s})$ of the observer program is relatively small. 


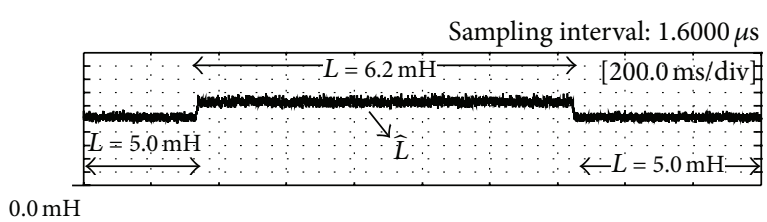

(a) The estimated inductance $\widehat{L}(1.0 \mathrm{mH} /$ div $)$

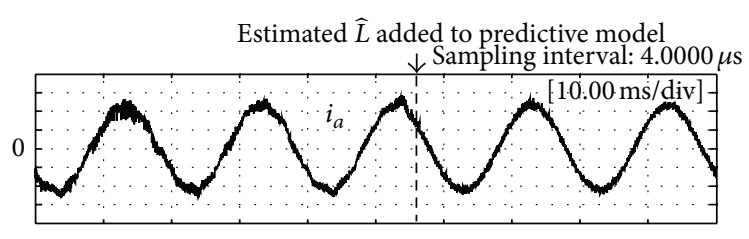

(b) The change of the phase-a current $i_{a}(3.0 \mathrm{~A} / \mathrm{div})$ when the inductance observer begins to work

FIGURE 7: Experimental waveforms of the inductance observer.

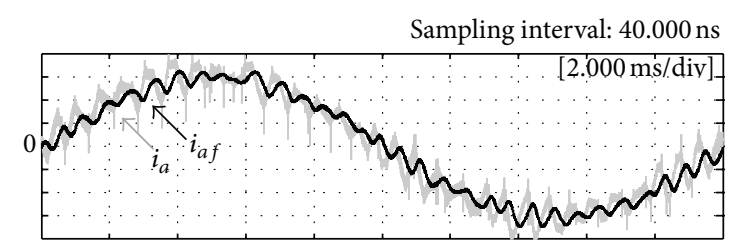

(a)

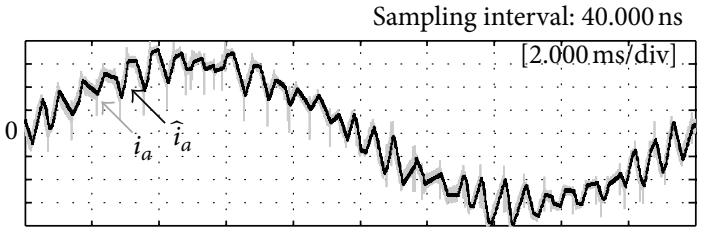

(b)

FIGURE 8: Experimental waveforms (2.0 A/div) of the filter time delay observer in the intense background noises environment.

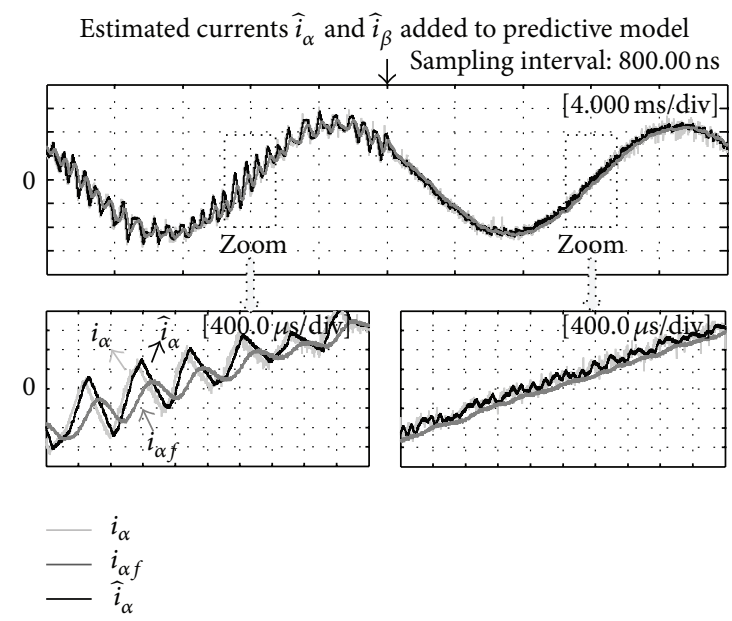

(a) Waveforms of $\alpha$-axis currents $i_{\alpha}, i_{\alpha f}$, and $\hat{i}_{\alpha}(3.0 \mathrm{~A} / \mathrm{div})$ before and after the adding of the estimated results
Estimated currents $\hat{i}_{\alpha}$ and $\hat{i}_{\beta}$ added to predictive model $\downarrow$ Sampling interval: $400.00 \mathrm{~ns}$

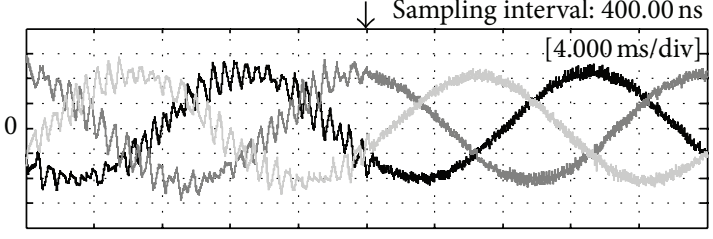

(b) Three-phase currents $i_{a}, i_{b}$, and $i_{c}(3.0 \mathrm{~A} / \mathrm{div})$ before and after the adding of the estimated results

FIGURE 9: Experimental waveforms of the filter time delay observer.

\section{Conclusions}

A three-phase power converter system based on FCS-MPC is built in this paper. Considering existing model parameter error and filter time delay in the FCS-MPC strategy, this paper analyzes the impacts of the input inductance error and filter time delay, respectively. The input inductance error and filter time delay produced obvious impacts on the performance of power converters.

The designed model-based observer can estimate the actual value of inductance and the currents without filter time delay. With the addition of the observer, the robustness and performance of FCS-MPC strategy have been improved. The experimental results show that, compared with the traditional
FCS-MPC algorithm, the proposed algorithm can effectively eliminate the influences produced by inductance error and filter time delay, such as the distortion and harmonic of the input current. The proposed FCS-MPC based power converter has lower current THD and more concentrated switching frequency distribution with the addition of the observer. The cost time of the observer program is relatively small. These aspects are good for the practical implementation.

\section{Conflict of Interests}

The authors declare that there is no conflict of interests regarding the publication of this paper. 


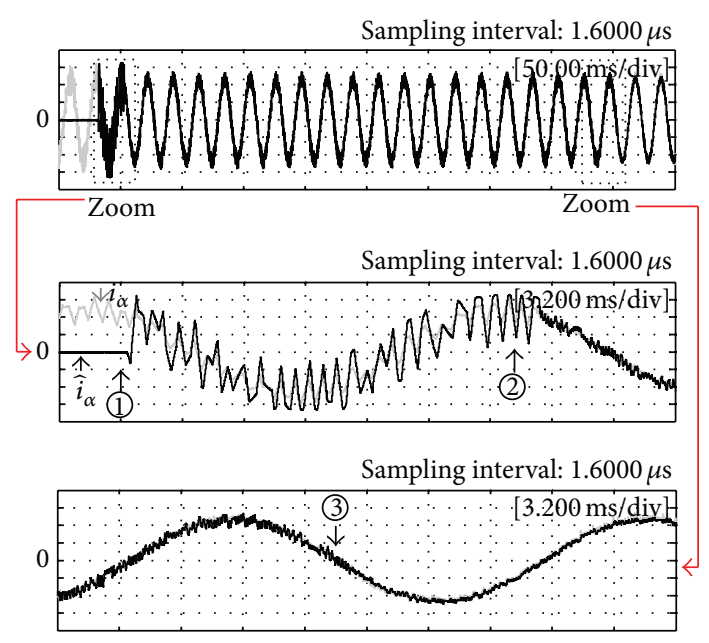

(a)

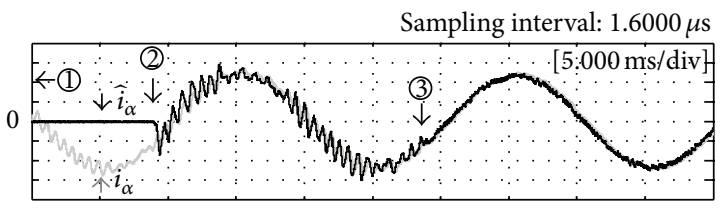

(b)

FIGURE 10: Waveforms of the currents $i_{a}$ and $\hat{i}_{a}(3.0 \mathrm{~A} / \mathrm{div})$ with different startup sequence of the observers. (a) Filter time delay observer starts first, then the inductance observer starts (1): filter time delay observer starts; (2): the estimated values of filter time delay observer are passed to the current prediction of FCS-MPC; and (3): the inductance observer starts and passes its estimated value to the current prediction of FCS-MPC). (b) Inductance observer starts first, and then the filter time delay observer starts (1): the inductance observer starts and passes its estimated value to the current prediction of FCS-MPC; (2): filter time delay observer starts; and (3): the estimated values of filter time delay observer are passed to the current prediction of FCS-MPC).

\section{Acknowledgments}

The authors are grateful to the financial support from the National Key Basic Research Program of China (973 Program) under Grant 2013CB035600, the National Natural Science Foundation of China under Grant 51307121, and the Key Technologies Research and Development Program of Tianjin under Grant 13ZCZDGX01100.

\section{References}

[1] K.-H. Chao and C.-T. Hsieh, "Mathematical modeling and fault tolerance control for a three-phase soft-switching mode rectifier," Mathematical Problems in Engineering, vol. 2013, Article ID 598130, 13 pages, 2013.

[2] J. Tlustý, J. Škramlík, J. Švec, and V. Valouch, "Analytical modeling and simulation of four-switch hybrid power filter working with sixfold switching symmetry," Mathematical Problems in Engineering, vol. 2012, Article ID 292178, 17 pages, 2012.

[3] L. Ge, X. Yuan, and Z. Yang, "Control system design of shunt active power filter based on active disturbance rejection and repetitive control techniques," Mathematical Problems in Engineering, vol. 2014, Article ID 864989, 6 pages, 2014.

[4] J. R. Rodríguez, J. W. Dixon, J. R. Espinoza, J. Pontt, and P. Lezana, "PWM regenerative rectifiers: state of the art," IEEE Transactions on Industrial Electronics, vol. 52, no. 1, pp. 5-22, 2005.

[5] S. Thielemans, T. J. Vyncke, and J. Melkebeek, "Weight factor selection for model-based predictive control of a four-level flying-capacitor inverter," IET Power Electronics, vol. 5, no. 3, pp. 323-333, 2012.

[6] K. G. Pavlou, M. Vasiladiotis, and S. N. Manias, "Constrained model predictive control strategy for single-phase switch-mode rectifiers," IET Power Electronics, vol. 5, no. 1, pp. 31-40, 2012.
[7] M. Rivera, C. Rojas, J. Rodriguez, and J. Espinoza, "Methods of source current reference generation for predictive control in a direct matrix converter," IET Power Electronics, vol. 6, no. 5, pp. 894-901, 2013.

[8] J. Rodriguez, M. P. Kazmierkowski, J. R. Espinoza et al., "State of the art of finite control set model predictive control in power electronics," IEEE Transactions on Industrial Informatics, vol. 9, no. 2, pp. 1003-1016, 2013.

[9] J. Scoltock, T. Geyer, and U. K. Madawala, "A comparison of model predictive control schemes for MV induction motor drives," IEEE Transactions on Industrial Informatics, vol. 9, no. 2, pp. 909-919, 2013.

[10] R. P. Aguilera, P. Lezana, and D. E. Quevedo, "Finite-controlset model predictive control with improved steady-state performance," IEEE Transactions on Industrial Informatics, vol. 9, no. 2, pp. 658-667, 2013.

[11] V. Yaramasu, M. Rivera, B. Wu, and J. Rodriguez, "Model predictive current control of two-level four-leg inverters-part I: concept, algorithm, and simulation analysis," IEEE Transactions on Power Electronics, vol. 28, no. 7, pp. 3459-3468, 2013.

[12] P. Cortes, A. Wilson, S. Kouro, J. Rodriguez, and H. Abu-Rub, "Model predictive current control of two-level four-leg inverters-part II: experimental implementation and validation," IEEE Transactions on Power Electronics, vol. 28, no. 7, pp. 34693478, 2013.

[13] P. E. Kakosimos and A. G. Kladas, "Implementation of photovoltaic array MPPT through fixed step predictive control technique," Renewable Energy, vol. 36, no. 9, pp. 2508-2514, 2011.

[14] H. A. Young, M. A. Perez, J. Rodriguez, and H. Abu-Rub, "Assessing finite-control-set model predictive control: a comparison with a linear current controller in two-level voltage source inverters," IEEE Industrial Electronics Magazine, vol. 8, no. 1, pp. 44-52, 2014.

[15] S. Kouro, P. Cortés, R. Vargas, U. Ammann, and J. Rodríguez, "Model predictive control-a simple and powerful Method 
to control power converters," IEEE Transactions on Industrial Electronics, vol. 56, no. 6, pp. 1826-1838, 2009.

[16] J. Rodríguez, J. Pontt, C. Silva et al., "Predictive current control of a voltage source inverter," IEEE Transactions on Industrial Electronics, vol. 54, no. 1, pp. 495-503, 2007.

[17] P. Cortés, J. Rodríguez, P. Antoniewicz, and M. Kazmierkowski, "Direct power control of an AFE using predictive control," IEEE Transactions on Power Electronics, vol. 23, no. 5, pp. 2516-2523, 2008.

[18] P. Cortes, J. Rodriguez, C. Silva, and A. Flores, "Delay compensation in model predictive current control of a three-phase inverter," IEEE Transactions on Industrial Electronics, vol. 59, no. 2, pp. 1323-1325, 2012. 


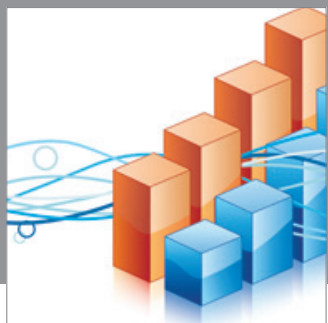

Advances in

Operations Research

mansans

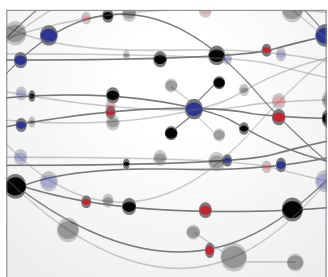

The Scientific World Journal
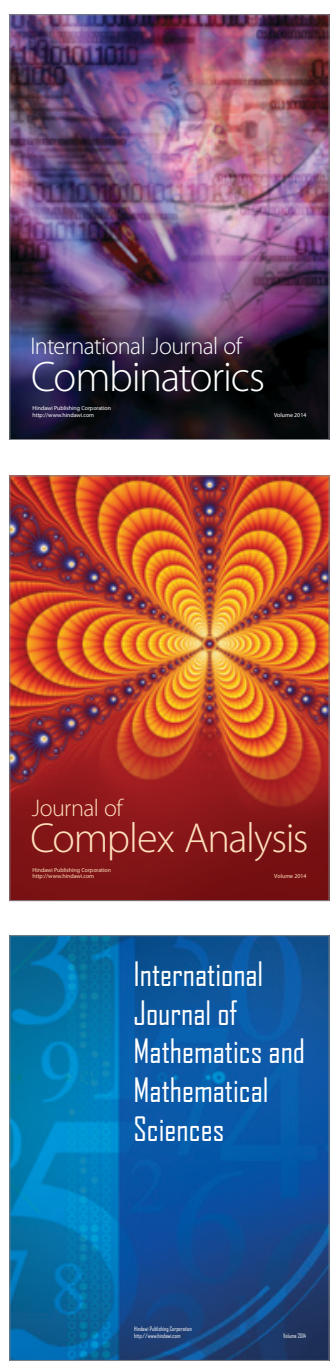
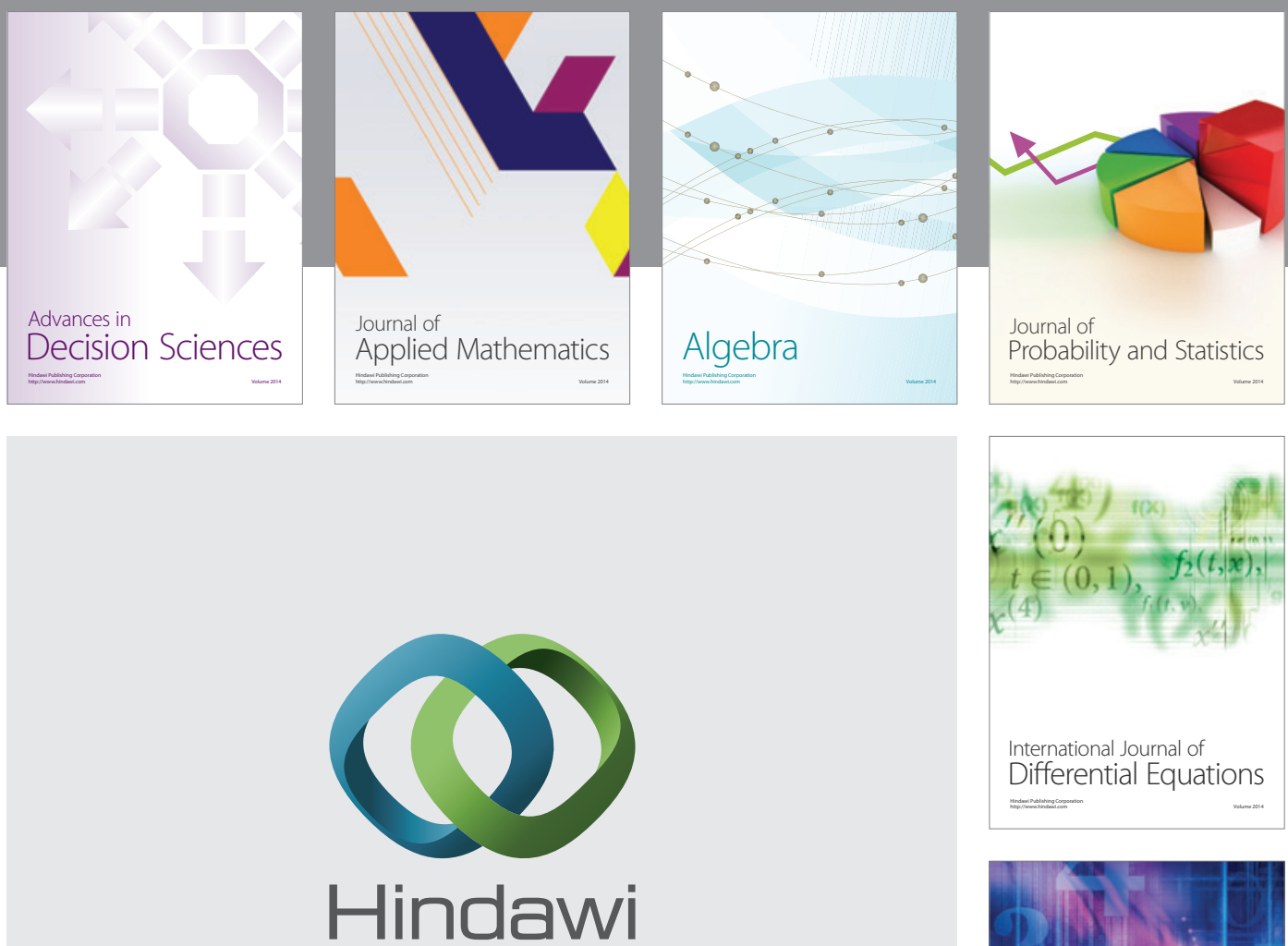

Submit your manuscripts at http://www.hindawi.com
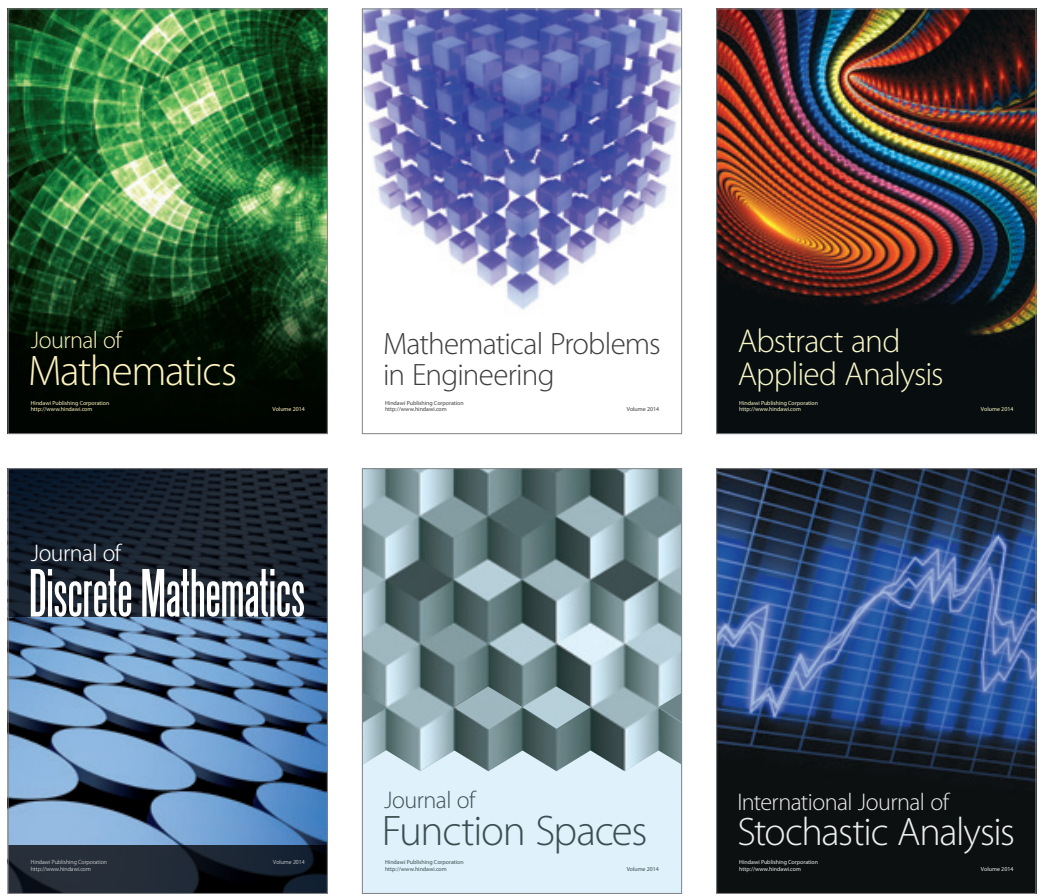

Journal of

Function Spaces

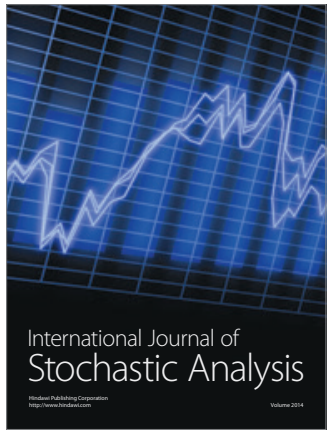

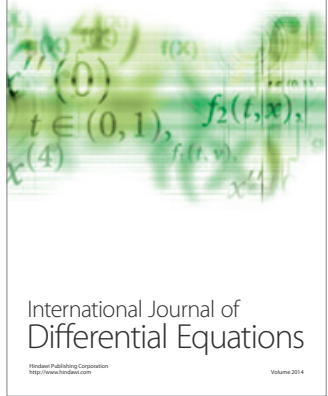
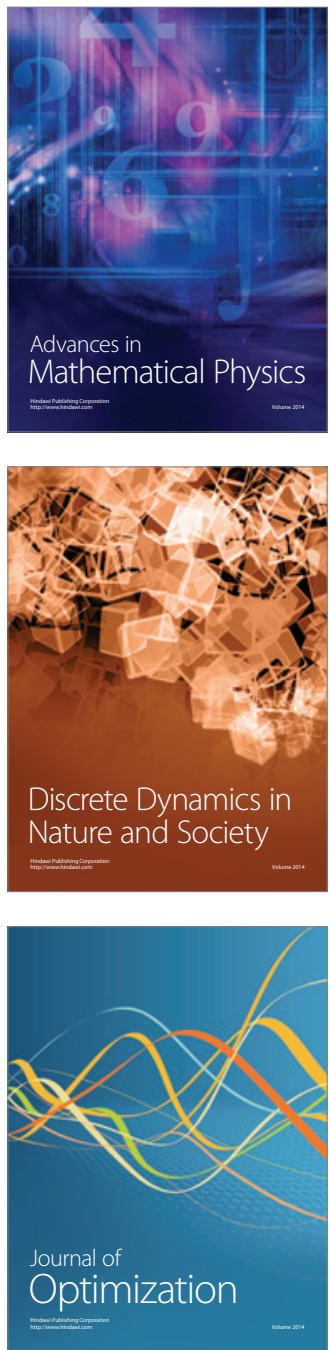